\title{
Synthesis and Purification of Peptide Nucleic Acids
}

Peptide nucleic acids (PNAs) are DNA analogs in which the normal phosphodiester backbone is replaced by 2-aminoethyl glycine linkages (Fig. 4.11.1). In spite of this significant chemical modification, the hybridization of PNAs with DNA or RNA follows normal rules for Watson-Crick pairing and occurs with high affinity. The fact that PNAs possess a dramatically different backbone and bind efficiently has created enormous interest in their application.

This unit describes the synthesis and purification of PNAs. PNAs can be synthesized using either automated procedures (see Basic Protocol 1; Mayfield and Corey, 1999) or manual procedures (see Basic Protocol 2; Norton et al., 1995). The goal here is to provide the reader with the basic information necessary to initiate experiments with PNAs.

NOTE: It is essential that the reagents used for PNA synthesis, whether automated or manual, be kept as anhydrous as possible, because contamination with water will result in incomplete coupling of monomers during synthesis.

\section{AUTOMATED SYNTHESIS OF PEPTIDE NUCLEIC ACIDS}

The automated synthesis of PNAs is illustrated in Figure 4.11.2. Automated synthesis is a convenient strategy for obtaining PNAs, and is performed using the Expedite synthesizer (Applied Biosystems). This instrument has been specially adapted for PNA synthesis, with proprietary software for FMOC chemistry instead of the phosphoramidite chemistry used for typical oligonucleotide synthesis. It is probably possible to adapt other synthesizers for PNA synthesis; however, the investigator will need to consider whether the number of PNAs needed justifies the time and expense required to adapt the instrumentation.

The reagents used are available from Applied Biosystems separately or as an Expedite PNA kit. Two resins are available: a xanthen alkonic acid (XAL) resin and a peptide amide linker (PAL) resin. Although either is adequate for automated synthesis, the XAL resin is preferred because the cleavage step is considerably shorter (5 min according to Applied Biosystems, compared to 90 min for the PAL resin).

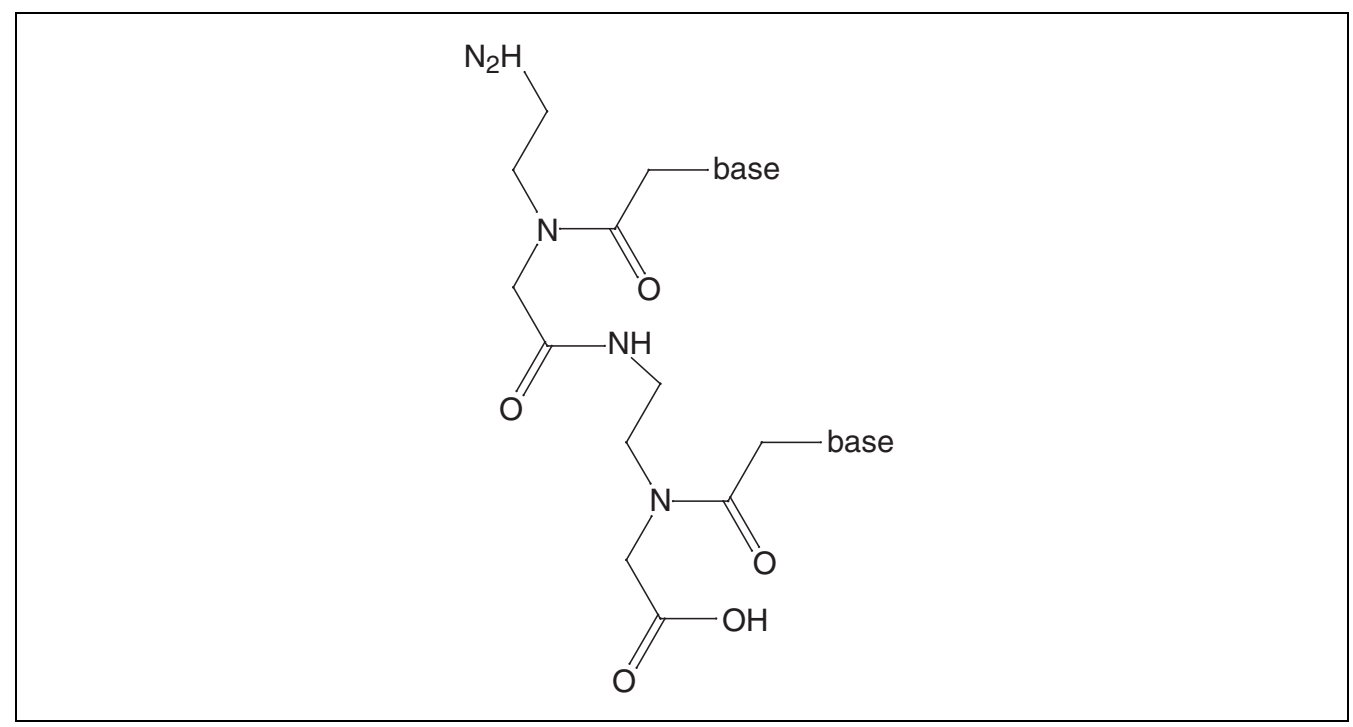

Figure 4.11.1 Structure of a peptide nucleic acid.

Contributed by Dwaine A. Braasch, Christopher J. Nulf, and David R. Corey

Current Protocols in Nucleic Acid Chemistry (2002) 4.11.1-4.11.18

Copyright $\odot 2002$ by John Wiley \& Sons, Inc.
BASIC

PROTOCOL 1

Synthesis of Modified

Oligonucleotides and Conjugates

\subsection{1}

Supplement 9 


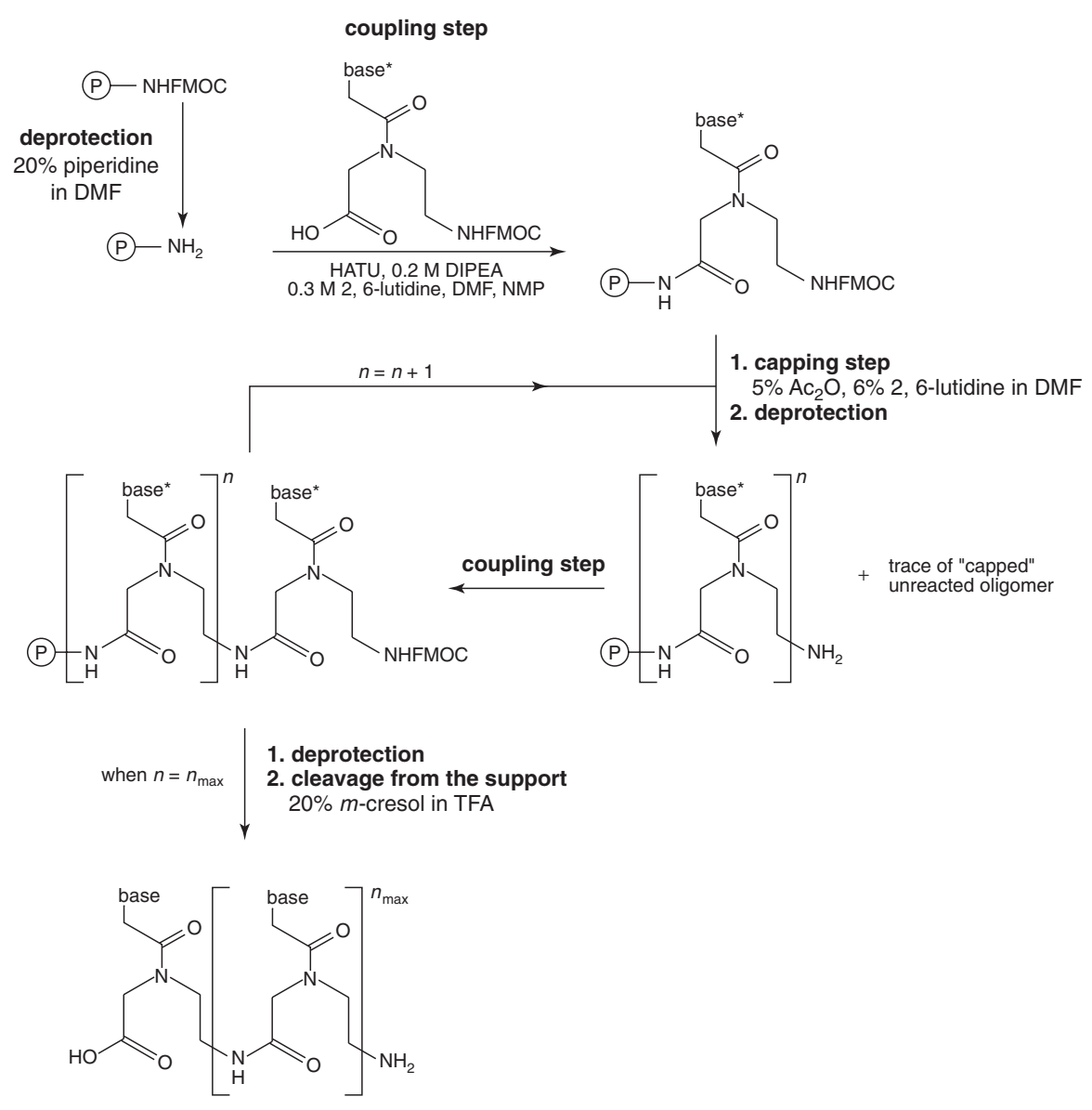

PNA monomers
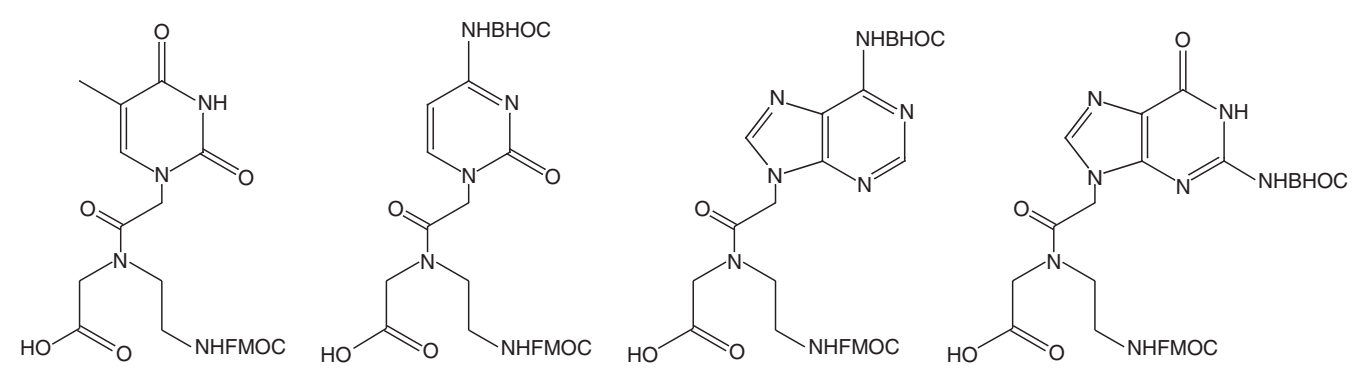

Figure 4.11.2 Automated PNA synthesis as described in Basic Protocol 1. Abbreviations: $\mathrm{Ac}_{2} \mathrm{O}$, acetic anhydride; base*, $\mathrm{N}$-protected nucleobase (see PNA monomers); BHOC, benzhydryloxycarbonyl; DIPEA, diisopropylethylamine; DMF, $N, N$ dimethylformamide; FMOC, 9-fluorenylmethoxycarbonyl; HATU, O-(7-azabenzotriazol-1-yl)-1,1,3,3-tetramethyluronium hexafluorophosphate; NMP, N-methylpyrrolidone; P, FMOC-XAL-PEG-polystyrene; TFA, trifluoroacetic acid.

2-Aminoethoxy-2-ethoxy acetic acid (AEEA) is used as a linker molecule for PNA synthesis. A linker molecule is used to introduce a controlled space between PNAs or between a PNA and some other functional or spatially addressable large group, such as rhodamine or biotin. Linked PNAs (bis-PNAs) possess an enhanced ability to bind by strand invasion and are an important tool for recognition of duplex DNA. Linker coupling is often inefficient, so it is always done two times.

Although the synthesis described here is an automated process, this does not excuse the experimenter from closely monitoring the various steps throughout the synthesis. Careful observation to ensure proper machine function can result in catching errors as they occur

Synthesis and Purification of Peptide Nucleic Acids and allow manual intervention that can rescue the synthesis and prevent loss of reagents and time (see Critical Parameters).

\subsection{2}




\section{Materials}

PNA Expedite reagents (Applied Biosystems)

FMOC-PNA monomers (Fig. 4.11.2): 9-fluorenylmethoxycarbonyl-protected peptide nucleic acid monomers (A, T, C, and G), base protected with benzhydryloxycarbonyl (BHOC)

Diluent: $N$-methylpyrrolidone (NMP)

Activator: 7-aza-1-hydroxybenzotriazole (HOAt) or

$O$-(7-azabenzotriazol-1-yl)-1,1,3,3-tetramethyluronium hexafluorophosphate (HATU)

Linker: 2-aminoethoxy-2-ethoxy acetic acid (AEEA)

Base solution: 0.2 M diisopropylethylamine (DIPEA)/0.3 M 2,6-lutidine

Deblocking solution: $20 \%(\mathrm{v} / \mathrm{v})$ piperidine in $N, N$-dimethylformamide (DMF)

Capping solution: 5\% (v/v) acetic anhydride/6\% (v/v) 2,6-lutidine in DMF

$N, N$-Dimethylformamide (DMF), anhydrous (Burdick Jackson; wash A is

Opti-Dry DMF from Fisher; wash B is anhydrous DMF from Applied

Biosystems)

Amino acids (Novabiochem, Advanced Chemtech)

Isopropyl alcohol (optional)

Cleavage cocktail: $20 \%$ (v/v) $m$-cresol (Sigma-Aldrich) in trifluoroacetic acid

(TFA; Burdick Jackson)

Diethyl ether, $-20^{\circ} \mathrm{C}$

Expedite 8909 synthesizer (Applied Biosystems)

FMOC-XAL-PEG-PS synthesis column $(0.2 \mu$ mol prepacked; Applied Biosystems)

10 -mL syringe

1.5-mL, 0.2- $\mu \mathrm{m}$ polytetrafluoroethylene (PTFE) or regenerated cellulose spin column (Millipore)

Additional reagents and equipment for automated synthesis (see manufacturer's instructions) and for purification and analysis of PNAs (see Basic Protocol 3)

NOTE: Powdered reagents such as monomers, activator, and linker (AEEA) should be unpacked on arrival and stored at $-20^{\circ} \mathrm{C}$ in a sealed container containing Drierite desiccant. Monomers should be inspected upon arrival. Clumps of reagent may indicate that water has been introduced during shipping.

NOTE: The authors use three sources of DMF because the bottles from Fisher (Opti-Dry) and Applied Biosystems fit onto the input fitting and reagent port on the synthesizer, and the third can be used following synthesis. All DMF must be anhydrous and should have a low amine content to reduce the likelihood of side reactions. DMF should be purchased in 100-mL volumes to ensure that it is used quickly, minimizing the likelihood that contaminating water will interfere with synthesis.

\section{Prepare reagents}

1. Dry all powdered reagents in vacuo overnight prior to solubilizing.

Although the authors have found that drying in vacuo is most necessary during humid weather, it is always a worthwhile precaution.

2. Warm amber bottles in which FMOC-PNA monomers were shipped to room temperature prior to solubilization to prevent water from condensing inside the container.

3. Solubilize FMOC-PNA monomers by adding $3.25 \mathrm{~mL}$ NMP diluent directly to each amber bottle (final $216 \mathrm{mM}$ monomer). Allow the mixture to sit undisturbed for 30 min at room temperature.

Synthesis of Modified Oligonucleotides and Conjugates

4.11.3

Supplement 8 
Premature vortexing of the monomer at this point will cause partially dissolved clumps of monomer to stick to the side of the bottle. If this occurs, extra time should be taken to bring the clumps to the bottom of the bottle. Typically, monomers $A, T$, and $G$ do not require vortexing, and a gentle swirling of the bottle's contents will suffice. Solubilization of monomer $C$ often requires some assistance. Intermittent heating of monomer $C$ in a shallow water bath at $37^{\circ} \mathrm{C}$ can assist in solubilization, as can occasional vortexing or sonication. The authors have also diluted the C monomer in an additional $0.5 \mathrm{~mL}$ of NMP and have observed that this facilitates dissolving the reagent without decreasing synthesis yield.

Experimenters should note that solubility can be dependent on the lot number of PNA monomer; some lots can be more or less difficult to dissolve than others.

The volumes indicated for reagent solubilization (steps 3 to 5) are adequate for 37 cycles, but the volumes prepared must ultimately accommodate the specific sequences and coupling events required for a given synthesis. Each cycle requires $90 \mu \mathrm{L}$ monomer, $90 \mu \mathrm{L}$ activator, $90 \mu \mathrm{L}$ base, $1.2 \mathrm{~mL}$ deblocking solution, $2.39 \mathrm{~mL} D M F$ wash A, $36 \mu \mathrm{L}$ DMF wash $B$, and $300 \mu L$ capping solution. These volumes are estimates. The investigator should always load significantly more reagent on the synthesizer to ensure that no reagent runs out during the final coupling steps.

4. Dissolve PNA activator (HOAt or HATU) in $13.5 \mathrm{~mL}$ anhydrous DMF (final 182 $\mathrm{mM})$.

Although HOAt is generally less expensive, it is also less efficient as a coupling reagent than HATU, which was developed specifically for PNA synthesis. HOAt should only be used for shorter sequences ( $\leq 12$ couplings). HATU has been used for syntheses requiring $>40$ couplings.

5. Dissolve AEEA linker in $2.4 \mathrm{~mL}$ NMP diluent (final $209 \mathrm{mM}$ ).

\section{Perform PNA synthesis}

6. Load the above reagents as well as base solution, amino acids, deblocking solution, and capping solution onto an Expedite 8909 synthesizer with an FMOC-XAL-PEGPS column.

Monomers, activator, and linker should not remain on the machine for $>2$ weeks. Amino acids should not be left on the machine for $>2$ days due to their tendency to crystallize. Amino acids are the least expensive reagent and it is cost effective to replace these solutions frequently rather than risk failed syntheses or damage to the instrument.

7. Perform synthesis according to manufacturer's programs and specifications, stopping before removal of the final FMOC group.

It is critical that the final FMOC be left on the PNA until a decision has been made to cleave the PNA from the resin or add another group. If synthesis is complete and the PNA is to be cleaved from the resin, proceed to step 9.

8. Optional: Perform any desired manual additions (e.g., see Support Protocols 1 and 2).

9. Implement the final deblock option in the prime menu of the Expedite PNA software. Cycle the final deblock procedure one time to complete the double deblock procedure.

\section{Wash and prepare PNA-bound resin}

10. Remove the column from the synthesizer and wash four times with $10 \mathrm{~mL}$ DMF, reversing the direction of flow through the column each time.

Synthesis and Purification of Peptide Nucleic Acids

4.11.4 
12. Dry the resin in vacuo for a minimum of $30 \mathrm{~min}$ or by blowing filtered house air across the column for 3 to $5 \mathrm{~min}$, reversing the ends frequently.

Resin is sufficiently dry when the resin plug slides easily from end to end as the air input is reversed.

\section{Cleave PNA from solid-phase resin}

13. Transfer the dried, resin-bound, deprotected PNA to a 1.5-mL, 0.2- $\mu \mathrm{m}$ PTFE or regenerated cellulose spin column.

14. Add $250 \mu \mathrm{L}$ cleavage cocktail and incubate $90 \mathrm{~min}$ at room temperature.

Although cleavage of PNAs from the FMOC-XAL-PEG-PS resin occurs in 5 min, cleavage of the protecting groups on the PNA side-chains requires at least 90 min. Complete cleavage of the BHOC side chains can be verified by MALDI-TOF mass spectrometry. Incomplete cleavage will result in a mass that exceeds the expected value by increments of the BHOC mass (100.1). Also, in the authors'experience, products that retain one or more protecting groups will have longer retention times by RP-HPLC (the protecting groups are hydrophobic).

Uniform cleavage is dependent on the freshness of the TFA, while the m-cresol serves as a molecular scavenger. The quality of the TFA can be checked by observing the color of the solution. It should be clear to slightly yellow. If the color progresses to a more orangebrown color, it should not be used for cleavage, but may still be used in preparing RP-HPLC buffer A (see Basic Protocol 3). In addition to solution color, TFA should release a small amount of fume (smoke) when the container is opened. If this is not observed, it should not be used for any procedure involving PNAs.

CAUTION: TFA is caustic and should be dispensed using only glass pipets or pipet tips containing a charcoal filter (Intermountain Scientific). The charcoal filter serves as a barrier that protects the pipettor seals from the TFA fumes.

15. Centrifuge $2 \mathrm{~min}$ at $1300 \times g$ for a PTFE filter or $2 \mathrm{~min}$ at $8400 \times g$ for a regenerated cellulose filter.

16. Repeat steps 14 and 15 , but reduce cleavage time to $5 \mathrm{~min}$.

17. Collect the cleavage filtrate, remove the filter unit, and precipitate the PNA by adding $1 \mathrm{~mL}$ cold $\left(-20^{\circ} \mathrm{C}\right)$ diethyl ether. Invert the tube several times to ensure complete precipitation.

If the synthesis has been successful, the precipitated PNA should be obvious.

18. Centrifuge precipitated PNA $2 \mathrm{~min}$ at $1300 \times g$. Discard the supernatant.

19. Wash the pellet three times with $1 \mathrm{~mL}$ diethyl ether, vortexing to suspend the pellet.

20. Centrifuge $2 \mathrm{~min}$ at $8400 \times g$ to repack the pellet.

Centrifugation speeds of more than $8400 \times g$ should be avoided because tight packing of PNA makes it difficult to dissolve the pellet.

21. Remove as much of the supernatant as possible by aspiration and then air dry the pellet for 5 to $10 \mathrm{~min}$ in a chemical fume hood.

22. Hydrate the pellet with $200 \mu \mathrm{L}$ sterile water (for a $2-\mu \mathrm{mol}$ synthesis) and allow the tube to remain undisturbed for 10 to $15 \mathrm{~min}$ at $65^{\circ} \mathrm{C}$.

Only slight vortexing should be required to complete PNA solubilization.

23. Purify and analyze PNA (see Basic Protocol 3).

Synthesis of Modified Oligonucleotides and Conjugates

4.11.5 
SUPPORT

PROTOCOL 1

SUPPORT PROTOCOL 2

Synthesis and Purification of Peptide Nucleic Acids

\subsection{6}

\section{ADDING PEPTIDES TO PNAS}

The addition of peptide sequences to PNAs is a convenient method for obtaining conjugates in which the peptide domain enhances hybridization (Zhang et al., 2000) or cell uptake (Simmons et al., 1997) of the attached PNA . Peptides can be added to a PNA in a number of ways. If the peptide contains three or fewer different amino acids, it can be conveniently added immediately before or after automated synthesis using the three open ports on the Expedite 8909 synthesizer in addition to the four dedicated to PNA monomers. All amino acids should be double coupled since amino acid coupling is sometimes inefficient. Also, since amino acids are inexpensive relative to PNA monomers, generous use of amino acids during coupling is a cost-effective strategy for optimizing synthesis yields.

If more than three different amino acids need to be added, it is often more convenient to contract a dedicated peptide synthesis facility to add the completed peptide. In this case, it is recommended that the first amino acid of the peptide be coupled to the newly synthesized PNA prior to shipping the resin. The authors have found that this procedure reduces the likelihood of the $\mathrm{N}$ terminus becoming blocked during shipping and storage. The synthesis of the PNA should be coordinated with the facility that will add the peptide, so that delays between syntheses are avoided. Delay between syntheses can result in the spontaneous loss of FMOC groups, exposing the $\mathrm{N}$ terminus to modification and preventing its extension. The facility adding the peptide should be instructed to double or triple couple the first amino acid that is added at their facility. Prior to shipping, the column should be washed with DMF and dried in vacuo overnight.

The thiol group of cysteine provides a convenient reactive group for PNA modification. Additional care is required to adequately cleave PNAs or PNA conjugates containing sulfhydryl groups, as the authors and others (Goodwin et al., 1998) have noted that the sulfhydryl can be modified during deprotection and purification. To avoid modification of cysteine, the cleavage cocktail should be supplemented with $7.5 \mathrm{mg}$ pure crystalline phenol (Fisher) and $250 \mu \mathrm{L}$ ethanedithiol (Sigma-Aldrich) per $1 \mathrm{~mL}$ of cleavage cocktail. Cysteine-containing PNAs should be neutralized immediately after purification to avoid the formation of TFA adducts. Neutralization can be achieved by adding $0.5 \mathrm{~mL}$ of an aqueous solution of $0.1 \mathrm{M}$ ammonium acetate to the RP-HPLC fraction collection tube prior to collecting the PNA fraction. Incomplete neutralization will result in a product that is 97 mass units higher than expected, corresponding to an adduct with TFA.

\section{ADDITION OF BIOTIN}

Once a PNA is synthesized, fluorescent groups, biotin, or other labels can be added to the free $\mathrm{N}$ terminus prior to deprotection. The labeling of PNAs with biotin is described as an example.

\section{Additional Materials (also see Basic Protocol 1)}

Biotin (Sigma)

$42^{\circ} \mathrm{C}$ water bath

1 -mL syringe

1. Perform a normal automated PNA synthesis (see Basic Protocol 1, steps 1 to 8).

2. Dissolve $20 \mathrm{mg}$ biotin, $6.1 \mathrm{mg}$ HATU, and $1.4 \mathrm{mg}$ HOAt in $800 \mu \mathrm{l}$ DMF. Warm the solution to $42^{\circ} \mathrm{C}$ and vortex intermittently until completely dissolved.

Biotin is not readily soluble and will require warming and vortexing. Due to its poor solubility, biotin solutions should never be put on the synthesizer, as it will clog the lines.

3. Add $200 \mu \mathrm{L}$ PNA base solution and mix well. Allow components to activate for 5 $\min$ at $42^{\circ} \mathrm{C}$. 
4. Remove N-terminal FMOC group by performing the final two deblock cycles to remove the N-terminal cap of the resin-bound PNA (see Basic Protocol 1, step 9).

5. Wash the column two times from each end with $10 \mathrm{~mL}$ DMF.

6. Draw up the biotin solution into a 1-mL syringe and place into one end of the column.

7. Place a second syringe into the other end of the column and push the solution back and forth for $30 \mathrm{~min}$.

8. Repeat steps 5 to 7 with another 1-mL preparation of biotin solution.

Addition of biotin is repeated because of the inherently poor coupling efficiency of biotin.

9. Proceed to the procedure for cleavage of PNAs from solid-phase resin (see Basic Protocol 1, steps 10 to 23).

\section{MANUAL SYNTHESIS OF PEPTIDE NUCLEIC ACIDS}

The manual synthesis of PNAs is illustrated in Figure 4.11.3. Manual synthesis of PNAs is advantageous because PNAs can be obtained in larger amounts $(>2 \mu \mathrm{mol})$ than on the Expedite synthesizer. By changing the amount of resin used, one can prepare as much or as little PNA as needed for each experiment. Manual synthesis also avoids the need for a dedicated automated synthesizer. As with automated synthesis, it is important to keep reagents and materials as anhydrous as possible. However, the coupling reactions are more exposed to atmospheric water. The authors have found that syntheses that use tert-butyloxycarbonyl (BOC) monomers generally produce better yields than syntheses using FMOC monomers.

Manual PNA synthesis is often so efficient that a capping step can be dispensed with. It is sometimes advisable, however, to include a capping step after coupling of the monomer to simplify the HPLC purification. The individual experimenter will have to determine the necessity for capping. It is typically necessary for long syntheses or for syntheses that have failed in the past.

Many different types of apparatus can be used for manual peptide synthesis, and it is likely that these can be adapted for PNA synthesis. The apparatus described here (Fig. 4.11.4; Norton et al., 1995) uses common laboratory glassware and offers robust performance. While the details of manual synthesis will vary with apparatus, the outline of the procedure and the precautions that need to be taken will remain the same. Because a single missed step can ruin a labor-intensive synthesis, the authors follow a detailed checklist for each step. The checklist provides a written record that all steps were performed. A sample spreadsheet detailing the amount of reagent needed for synthesis is shown in Figure 4.11.5.

\section{Materials}

Nitrogen source

$N, N$-Dimethylformamide (DMF; OptiDry; Fisher)

4-Hydroxymethylphenylamidomethyl (PAM) resin protected with tert-butyloxycarbonyl (BOC; Applied Biosystems)

Carrier resin: PAM resin capped with an acetyl group (see Support Protocol 3)

BOC-PNA monomers (Fig. 4.11.3; Applied Biosystems):

tert-butyloxycarbonyl-protected peptide nucleic acid monomers (A, C, G, and

$\mathrm{T})$, base protected with benzyloxycarbonyl

2-(1H-Benzotriazol-1-yl)-1,1,3,3-tetramethyl uronium hexafluorophosphate

(HBTU) and 1-hydroxybenzotriazole (HOBt) activators (Applied Biosystems)

Fresh dichloromethane (DCM; Fisher)

$m$-Cresol

Trifluoroacetic acid (TFA; Burdick Jackson)

Synthesis of Modified

Oligonucleotides and Conjugates

4.11.7 


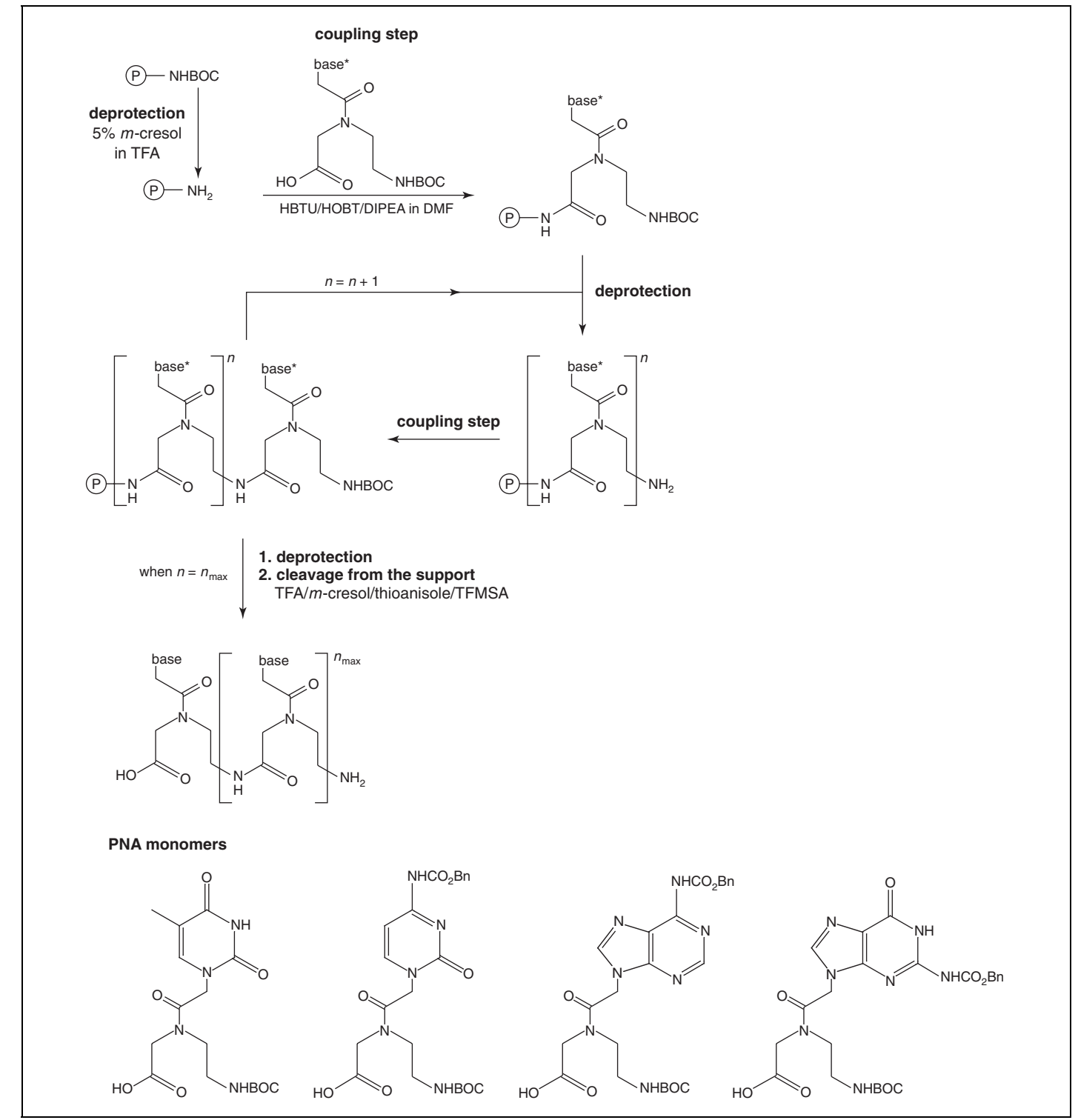

Figure 4.11.3 Manual PNA synthesis as described in Basic Protocol 2. Although a capping step can be added, it is often not required and is not shown here. Abbreviations: base*, $N$-protected nucleobase (see PNA monomers); BOC, tert-butyloxycarbonyl; Bn, benzyl; DIPEA, diisopropylethylamine; DMF, dimethylformamide; HBTU, 2-(1H-benzotriazol-1-yl)-1,1,3,3-tetramethyl uronium hexafluorophospate; HOBt, 1hydroxybenzotriazole; P, PAM resin; TFA, trifluoroacetic acid; TFMSA, trifluoromethanesulfonic acid.

Synthesis and Purification of Peptide Nucleic Acids
Pyridine

Diisopropylethylamine (DIPEA)

Methanol

Thioanisole

Trifluoromethanesulfonic acid (TFMSA; Aldrich)

Diethyl ether, ice cold

$250^{\circ} \mathrm{C}$ oven

$125-\mathrm{mL}$ vacuum filtration side-arm flasks

24/40 rubber septa

15-mL medium (C) fritted Pyrex funnel

Vacuum tubing

3-way valves

250-mL Wheaton bottles with caps

4.11.8 


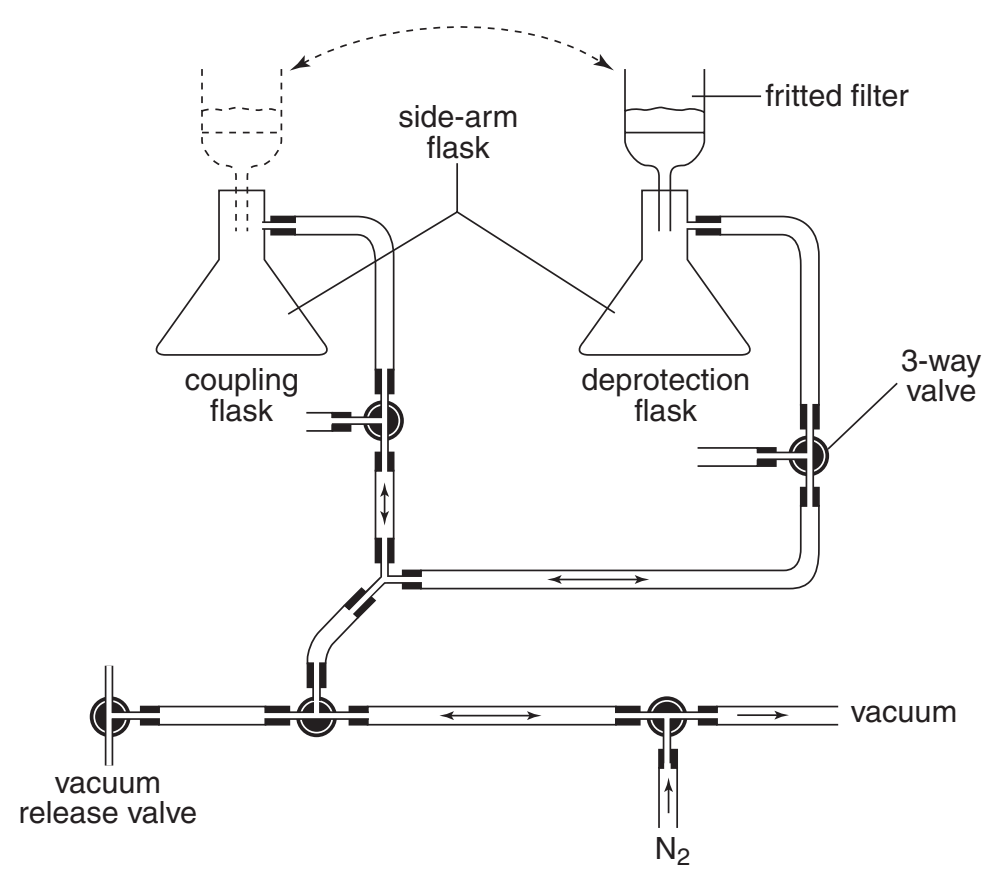

Figure 4.11.4 Apparatus for manual synthesis. Reprinted from Norton et al. (1995) with permission from Elsevier Science.

\begin{tabular}{|c|c|c|c|}
\hline & Monomer & $\begin{array}{c}\text { Molecular Weight } \\
\mathrm{g} / \mathrm{mol}\end{array}$ & $\begin{array}{l}\text { PNA mass after each coupling } \\
\mathrm{g} / \mathrm{mol}\end{array}$ \\
\hline C-Terminus & terminal amide & 17.00 & $\begin{array}{lll}- & \\
- & & l\end{array}$ \\
\hline 1 & Iysine & 128.00 & 145.00 \\
\hline 2 & A & 275.32 & 420.32 \\
\hline 3 & A & 275.32 & 695.64 \\
\hline 4 & C & 251.30 & 946.94 \\
\hline 5 & A & 275.32 & 1222.26 \\
\hline 6 & $\mathrm{G}$ & 291.32 & 1513.58 \\
\hline 7 & A & 275.32 & 1788.90 \\
\hline 8 & $T$ & 266.28 & 2055.18 \\
\hline 9 & $T$ & 266.28 & 2321.46 \\
\hline 10 & G & 291.32 & 2612.78 \\
\hline 11 & G & 291.32 & 2904.10 \\
\hline 12 & G & 291.32 & 3195.42 \\
\hline 13 & A & 275.32 & 3470.74 \\
\hline 14 & $T$ & 266.28 & 3737.02 \\
\hline \multicolumn{4}{|l|}{$\mathrm{N}$-Terminus } \\
\hline & & & Total Mass \\
\hline
\end{tabular}

\begin{tabular}{|c|c|}
\hline Amount of Resin Used & $10 \mathrm{mg}$ \\
\hline Substitution Number for Resin & $0.66 \mathrm{mmol} / \mathrm{g}$ \\
\hline Total Active Sites & $0.0066 \mathrm{mmol}$ \\
\hline PNA Mass & $3737.02 \mathrm{mg} / \mathrm{mmol}$ \\
\hline Amount of PNA to be Synthesized & $24.6 \mathrm{mg}$ \\
\hline
\end{tabular}

\begin{tabular}{|c|c|c|}
\cline { 2 - 3 } \multicolumn{1}{c|}{} & Equivalents & Amount Per Coupling \\
\hline A Monomer & 5.0 & $17.41 \mathrm{mg}$ \\
\hline G Monomer & 5.0 & $17.94 \mathrm{mg}$ \\
\hline C Monomer & 5.0 & $16.62 \mathrm{mg}$ \\
\hline T Monomer & 5.0 & $12.68 \mathrm{mg}$ \\
\hline HBTU & 4.5 & $11.26 \mathrm{mg}$ \\
\hline HOBt & 5.0 & $4.46 \mathrm{mg}$ \\
\hline DIPEA & 10.0 & $8.54 \mu \mathrm{L}$ \\
\hline DMF & - & Up to $1 \mathrm{~mL}$ \\
\hline
\end{tabular}

Figure 4.11.5 Example of a spreadsheet for the manual synthesis of a 14-base PNA including lysine at the $C$ terminus. The resin used produces a theoretical yield of $24.6 \mathrm{mg}$ of PNA upon cleavage.

Synthesis of Modified

Oligonucleotides and Conjugates

\subsection{9}

Supplement 8 
Lyophilizer

10 -mL flask with a ground glass joint

Desiccator

Additional reagents and equipment for purification and analysis of PNAs (see Basic Protocol 3)

NOTE: All powdered reagents such as monomers and activator should be unpacked on arrival and stored at $-20^{\circ} \mathrm{C}$ in a sealed container containing Drierite desiccant. Monomers should be inspected upon arrival. Clumps of reagent may indicate that water has been introduced during shipping. DMF should have a low amine content to reduce the likelihood of side reactions.

\section{Dry reagents and supplies}

1. Dry all monomers and coupling reagents overnight in vacuo before beginning synthesis.

To prevent accumulation of moisture, BOC-PNA monomers that have been refrigerated should be warmed to room temperature before opening.

2. Dry all glassware, pipet tips, and $1.5-\mathrm{mL}$ microcentrifuge tubes in a $250^{\circ} \mathrm{C}$ oven overnight prior to use. Flush pipet tips and microcentrifuge tubes with nitrogen to ensure they are dry prior to synthesis.

\section{Set up apparatus}

3. Clear a work area in a chemical hood.

4. Fit rubber septa over the tops of two $125-\mathrm{mL}$ side-arm flasks and make a single hole in the middle of each septum to allow a $15-\mathrm{mL}$ fritted funnel to be inserted without too much force.

5. Assemble the manual synthesis apparatus as in Figure 4.11.4 using vacuum tubing and 3-way valves such that the vacuum and nitrogen bubbling can be easily manipulated on and off.

6. Ensure that the $15-\mathrm{mL}$ fritted funnel is clean and unclogged. Pull $5 \mathrm{~mL} \mathrm{DMF}$ through the funnel to make sure it drains quickly.

\section{Prepare reagents}

7. In a 1.5-mL microcentrifuge tube, weigh out the appropriate amount of BOC-protected PAM resin and add enough carrier resin to give $\sim 50 \mathrm{mg}$ total resin weight.

The purpose of the carrier resin is to facilitate handling of the resin by allowing researchers to handle larger volumes.

The amount of PNA to be synthesized is dependent on the amount of resin used and the number of active sites on the resin. The number of active sites is based on the substitution number (Fig. 4.11.5), which should be listed on the resin bottle when purchased. The total number of active sites is based only on the amount the BOC-protected (noncarrier) PAM resin, as the carrier resin has been capped and contains no active sites.

PAM resin can also be purchased with the first amino acid already attached (Advanced Chemtech).

8. Load 1.5-mL microcentrifuge tubes with the proper amount of dry monomer for each coupling reaction. Use a 5-fold excess of monomer over the resin active sites.

Synthesis and Purification of Peptide Nucleic Acids

It is easiest to set up a spreadsheet (Fig. 4.11.5) with quantities of monomers, HBTU, HOBt, and DIPEA used for each activation/coupling reaction, checking them off during synthesis of the PNA. 
9. For each coupling reaction, measure out a 5-fold excess of HOBt (relative to resin active sites), a 4.5-fold excess of HBTU, 10 eq DIPEA, and 1 mL DMF. Draw DMF from the bottle using a syringe under nitrogen or argon gas to keep it as anhydrous as possible.

HOBt, HBTU, DIPEA, and DMF will be added to the monomer in each microcentrifuge tube just minutes before starting the coupling reaction (step 17). This method is the most efficient way of activating the monomers, and thus preventing deletions in the final product.

Aldrich sells DMF in Sure/Seal bottles, which have a rubber opening on a crimped lid so that a syringe can be inserted without uncapping the bottle and exposing the contents to air. A nitrogen-filled balloon may also be inserted through the rubber opening to continually flush the bottle with nitrogen during the synthesis.

10. Prepare $200 \mathrm{~mL}$ fresh 50:50 (v/v) DMF/DCM for washing. Also prepare $100 \mathrm{~mL}$ of $5 \%(\mathrm{v} / \mathrm{v}) \mathrm{m}$-cresol/TFA and $100 \mathrm{~mL}$ pyridine in separate clean, dry $250-\mathrm{mL}$ Wheaton bottles with caps.

The pyridine is used to wash the resin with the base prior to coupling.

CAUTION: TFA is caustic and should be dispensed using only glass pipets or pipet tips containing a charcoal filter (Intermountain Scientific). The charcoal filter serves as a barrier that protects the pipettor seals from the TFA fumes.

11. Assemble disposable glass pipets and bulbs, one for each reagent, and place near or on each reagent bottle.

For convenience and to prevent contamination, the authors usually tape a test tube onto each bottle as a pipet holder.

12. Place the $15-\mathrm{mL}$ fritted funnel into the deblocking flask. Wet the rubber septum and the funnel with a little methanol to help it slide in.

13. Add the dry resin to the $15-\mathrm{mL}$ fritted funnel without solubilizing it in DMF.

It is easier to get it all in this way.

\section{Perform synthesis}

14. Swell resin in $1 \mathrm{~mL} \mathrm{DMF}$ for $1 \mathrm{hr}$ with nitrogen bubbling.

15. Close and bleed vacuum line.

16. Add $1 \mathrm{~mL}$ of $5 \% \mathrm{~m}$-cresol/TFA to begin deprotection. Stir $3 \mathrm{~min}$ with nitrogen bubbling.

17. While the BOC group is being removed, activate (esterify) the first monomer to be added by adding HOBt, HBTU, DMF, and DIPEA (from step 9) to the monomer tube (step 8). Vortex until everything has gone into solution.

Typically, these solutions turn a tan color after vortexing. Failure to change color can sometimes be an indication that the activation step is not proceeding properly.

As the synthesis progresses, liquid will accumulate in the flasks. It is important to watch the level of liquids in the coupling and deprotection flasks and empty them when the volumes approach the fritted funnel. The deprotection flask can be emptied during a coupling step and vice versa.

18. Vacuum off deblocking solution from the resin.

19. Wash resin two times with $1 \mathrm{~mL}$ of 50:50 DMF/DCM.

20. Close and bleed vacuum line.

Synthesis of Modified Oligonucleotides and Conjugates

\subsubsection{1}

Supplement 8 
21. Remove funnel from the deprotection flask and insert into the rubber septum over the coupling flask.

22. Add activated monomer to resin and mix 20 min, with nitrogen bubbling, to couple the monomer to the resin (or growing PNA).

23. Vacuum off solution.

24. Wash six times with $1 \mathrm{~mL}$ of 50:50 DMF/DCM.

25. Wash with $1 \mathrm{~mL}$ pyridine then with $1 \mathrm{~mL}$ dry DMF.

If capping is necessary, add $1 \mathrm{~mL}$ of $1: 1(\mathrm{v} / \mathrm{v})$ acetic anhydride/DMF to the resin and mix for 20 min. Wash well with DMF and proceed to the next step.

26. Vacuum off solution.

27. Close and bleed vacuum line.

28. Remove funnel from the coupling flask and insert into the septum over the deblocking flask.

29. Repeat steps 16 to 28 for remaining monomer additions.

30. Remove the final protecting group as in steps 15 and 16.

31. Vacuum off deblocking solution.

32. Wash six times with $1 \mathrm{~mL}$ of 50:50 DMF/DCM.

33. Wash six times with $1 \mathrm{~mL}$ methanol.

34. Lyophilize overnight.

When the synthesis is complete, it is customary to wash the resin with methanol and to dry in vacuo overnight before the cleavage is carried out.

If the synthesis takes $>1$ day, it will be necessary to store the resin. Stop the synthesis just prior to deblocking the PNA, leaving the protecting group on. Wash the resin several times with HPLC-grade methanol. Remove the funnel with the resin from the coupling flask, put a Kimwipe over the top, and secure it with a rubber band. Label the funnel and put it in a desiccator. Resume the synthesis the following day by starting with swelling of the resin in NMP and deblocking the PNA (steps 14 to 16).

\section{Cleave PNA from resin}

35. Connect a clean $125-\mathrm{mL}$ vacuum flask to the synthesis apparatus in place of the coupling flask.

36. Attach a rubber septum over the top of the flask and insert the funnel that contains the resin.

37. Prepare $4 \mathrm{~mL}$ cleavage cocktail in a clean, dry $10-\mathrm{mL}$ flask that has a ground glass joint. Mix 1 part $m$-cresol, 1 part thioanisole, and 6 parts TFA. Cap the flask, put on gloves and a shield, crack open a vial of TFMSA, and add 2 parts TFMSA.

Typically, the cleavage cocktail turns brown at this point.

CAUTION: TFMSA is corrosive and extremely destructive to mucous membranes, the upper respiratory tract, eyes, and skin. Avoid skin contact and inhalation. Always use suitable protection.

38. Using a pipettor, add $1 \mathrm{~mL}$ cleavage cocktail to the resin. Using a very small flow of nitrogen into the flask, allow solution to barely bubble for $\sim 1 \mathrm{hr}$.

Synthesis and

Purification of

Peptide Nucleic

Acids

4.11.12

Supplement 8

Current Protocols in Nucleic Acid Chemistry 
39. Turn off the nitrogen and apply a vacuum to pull the cleavage solution into the clean flask. Remove as much of the cleavage solution as possible before proceeding to the precipitation.

Excess TFA will make it more difficult to precipitate the PNA. Excess TFA can be removed from the solution by blowing a steady stream of nitrogen into the flask until most of the solution is gone, or by applying a vacuum to the flask for several minutes.

40. With most of the cleavage solution gone, add $\geq 45 \mathrm{~mL}$ ice-cold diethyl ether to the flask and set it on ice.

41. Take a spatula and scrape the sides of the flask to loosen all of the PNA into solution.

There should be a white precipitate in the solution.

42. Pour the PNA/diethyl ether solution into a $50-\mathrm{mL}$ conical centrifuge tube, cap tightly, and centrifuge 3 min at $1040 \times g$ (e.g., 2500 rpm in a Beckman S4180 swinging bucket rotor), $5^{\circ} \mathrm{C}$, pelleting the PNA to the bottom of the tube.

43. Carefully decant or aspirate off the diethyl ether.

44. Wash pellet three times by adding $50 \mathrm{~mL}$ ice-cold diethyl ether to the tube, vortexing, centrifuging, and removing the supernatant.

45. After decanting the last time, place a Kimwipe over the tube containing the wellwashed pellet and place it in a desiccator. Attach the house vacuum and allow PNA to dry overnight.

46. Purify and analyze PNA (see Basic Protocol 3).

The PNA can be stored at $-20^{\circ} \mathrm{C}$ as a lyophilized product or as an aqueous stock solution (in deionized water). Traces of TFA will slightly acidify the solution and readily dissolve the PNA.

\section{PREPARATION OF CARRIER RESIN FOR MANUAL PNA SYNTHESIS}

Carrier resin is prepared by deprotecting a simple BOC-protected PAM resin and performing a capping step using acetic anhydride.

Additional Materials (also see Basic Protocol 2)

tert-Butyloxycarbonyl-protected 4-hydroxymethylphenylamidomethyl resin (e.g., BOC-Ala-PAM, BOC-Val-PAM, BOC-Ile-PAM; Applied Biosystems)

Acetic anhydride

HPLC-grade dichloromethane

HPLC-grade methanol

1. Set up the manual synthesis apparatus (Fig. 4.11.4) with a single flask.

2. Place $\sim 2 \mathrm{~g}$ BOC-protected PAM resin in the fritted funnel and swell with $1 \mathrm{~mL} \mathrm{DMF}$ for $1 \mathrm{hr}$.

3. Vacuum off DMF.

4. Add a large excess (e.g., $1 \mathrm{~mL}$ ) of $5 \%(\mathrm{v} / \mathrm{v}) \mathrm{m}$-cresol/TFA and deprotect the resin for 10 min with bubbling.

5. Wash two times with $1 \mathrm{~mL}$ DMF and repeat deprotection two more times.

The resin should be fully deprotected at this point.

6. Wash five times with $1 \mathrm{~mL} \mathrm{DMF}$ and once with $1 \mathrm{~mL}$ pyridine.

7. Add $1 \mathrm{~mL}$ of 1:1: (v/v) acetic anhydride/DMF and bubble for $20 \mathrm{~min}$.

8. Wash once with $1 \mathrm{~mL} \mathrm{DMF}$ and repeat step 7.

Synthesis of Modified Oligonucleotides and Conjugates

\subsubsection{3}


BASIC PROTOCOL 3

9. Wash once each with $1 \mathrm{~mL}$ DMF, $1 \mathrm{~mL}$ dichloromethane, and $1 \mathrm{~mL}$ methanol.

10. Dry overnight in a desiccator.

The resin can be prepared in bulk and stored at $4^{\circ} \mathrm{C}$ in the desiccator.

\section{PURIFICATION AND ANALYSIS OF PEPTIDE NUCLEIC ACIDS}

PNAs can be purified by reversed-phase HPLC (RP-HPLC) followed by matrix-assisted laser desorption/ionization time-of-flight mass spectrometry (MALDI-TOF-MS) (Fig. 4.11.6). PNAs are not, however, purified or analyzed by standard procedures used for oligonucleotides. PNA purification and analysis is more similar to that for peptides.

\section{Materials}

PNA sample solution (see Basic Protocols 1 and 2)

RP-HPLC buffer A: $0.1 \%$ (v/v) trifluoroacetic acid (TFA; Burdick Jackson) in water, passed through a 47-mm, 0.4- $\mu$ m nylon membrane (Whatman)

RP-HPLC buffer B: $0.1 \%$ (v/v) TFA in acetonitrile (Optima grade; Fisher), filtered through an Anodisc 47 filter $(0.22-\mu \mathrm{m}$; Whatman)

$\alpha$-Cyano-4-hydroxycinnamic acid (Sigma)

Isopropanol

High-performance liquid chromatograph (HPLC) with C18 reversed-phase column (300- $\AA$ Microsorb-MV column; Varian Analytical Instruments)

Matrix-assisted laser desorption/ionization time-of-flight (MALDI-TOF) mass spectrometer (Voyager-DE workstation; Applied Biosystems)

Lyophilizer

UV spectrophotometer

Additional reagents and equipment for HPLC and MALDI-TOF-MS

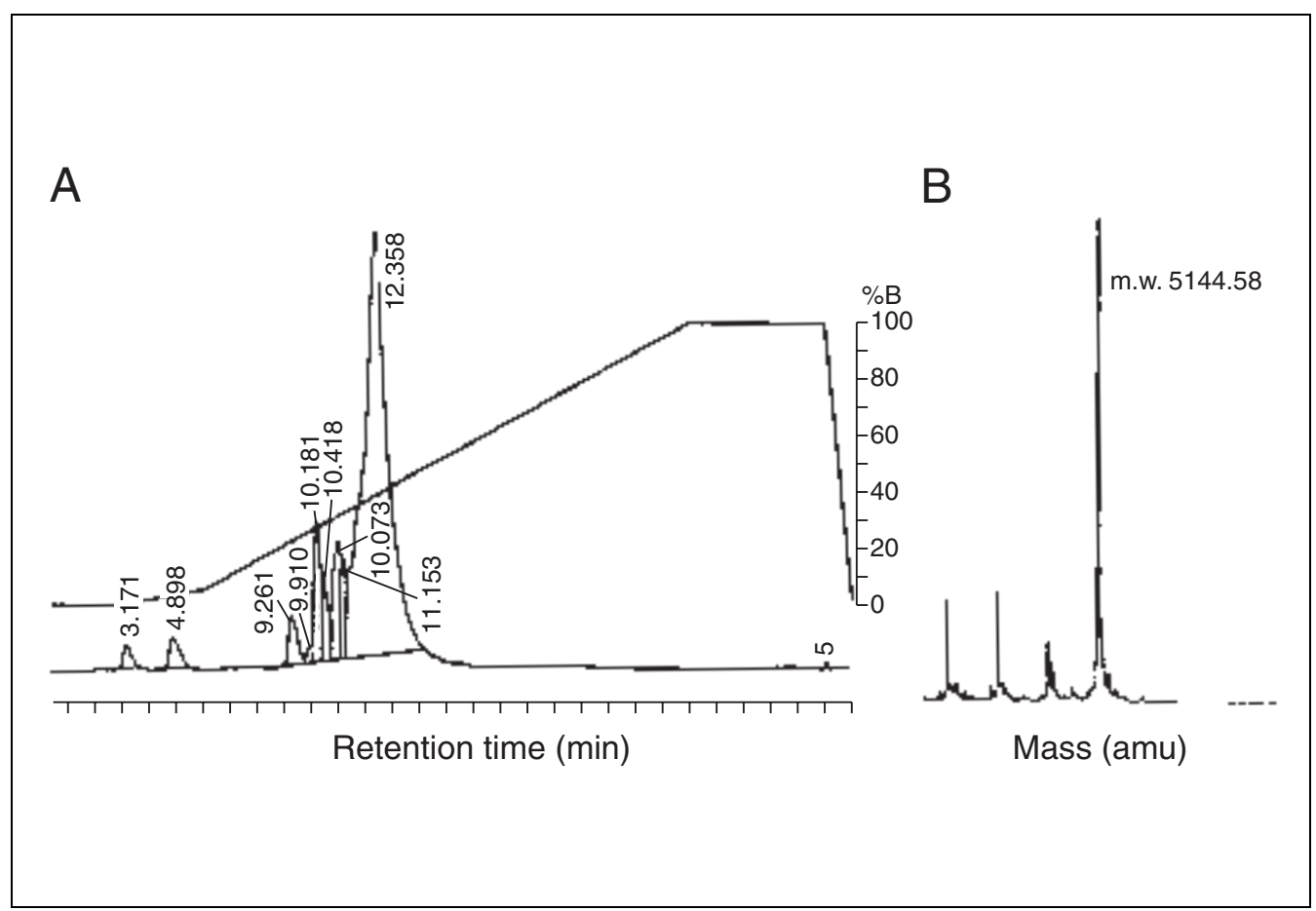

Figure 4.11.6 Typical (A) HPLC and (B) MALDI-TOF mass spectrometry data.

Synthesis and Purification of Peptide Nucleic

Acids

\subsubsection{4}


Purify by HPLC

1. Centrifuge a PNA solution $3 \mathrm{~min}$ at $12,000 \times g$, room temperature, to remove particulate.

2. Heat a $\mathrm{C} 18$ reversed-phase HPLC column to $55^{\circ} \mathrm{C}$.

PNAs tend to form internal structure or higher order aggregates; sharper peaks will often be obtained if the column is maintained at $55^{\circ} \mathrm{C}$ using a heated water jacket.

3. Set up a gradient of $0 \%$ to $5 \%$ (v/v) RP-HPLC buffer B in buffer A for 6 min followed by $5 \%$ to $100 \%$ buffer B in buffer A for 24 min.

4. Inject sample.

5. Collect fractions corresponding to major peaks.

\section{Analyze by MALDI-TOF-MS}

6. Spot a 1- $\mu$ L aliquot of each HPLC fraction on the laser target of a MALDI-TOF mass spectrometer.

7. Overlay the sample with $1 \mu \mathrm{L}$ matrix consisting of $10 \mathrm{mg} / \mathrm{mL} \alpha$-cyano-4-hydroxycinnamic acid in 25:75 (v/v) RP-HPLC buffers A/B.

8. Activate the laser and collect data.

Resolution is best when lower laser energies are used $(\sim 1400 \mathrm{mV})$ but higher energies are sometimes necessary, especially with long PNAs and PNA-peptide conjugates.

Analytical scale analysis by HPLC or mass spectral analysis of the crude product often reveals that only one product has been formed. If this is the case, the PNA can be conveniently purified by Sep-Pak Vac 6-mL (1 g) C18 cartridge (Waters Chromatography). Alternatively, PNAs can be purified by preparative C18 HPLC (Baker Bond, J.T. Baker). PNAs typically elute between $32 \%$ and $37 \%$ RP-HPLC buffer B and are routinely $>80 \%$ full-length material.

\section{Solubilize and store PNA}

9. Pool collected fractions containing the PNA with the appropriate mass. Freeze in an isopropanol/dry ice bath and lyophilize.

PNAs that are not to be used immediately can be stored indefinitely in a lyophilized form at $-20^{\circ} \mathrm{C}$ in a sealed storage box containing desiccant.

10. Dissolve the resulting pellet in $200 \mu \mathrm{L}$ sterile water and allow the tube to sit 5 to 10 min undisturbed at room temperature.

PNAs are readily soluble in aqueous solutions at pH 5.0 to 6.0 at high concentrations (millimolar) but are less soluble at higher $\mathrm{pH}$ 's. Solubility at neutral pH can be enhanced by the incorporation of charged amino acid residues at the termini of PNAs during synthesis. Heating solutions containing PNAs can also enhance solubility, and the authors recommend always heating PNAs to $>50^{\circ} \mathrm{C}$ immediately prior to use to ensure that aggregation is minimized.

The authors have repeatedly frozen and thawed solubilized PNAs without observing diminished hybridization, although for long-term storage it is best to store PNAs in lyophilized form.

\section{Analyze by $U V$ spectrophotometry}

11. Heat PNA stock solutions $5 \mathrm{~min}$ at $65^{\circ} \mathrm{C}$ to reduce aggregation prior to dilution and measurement.

PNAs readily aggregate because they are relatively hydrophobic. Heating is a convenient way to ensure that the measured concentration reflects the total concentration of PNA in the sample. Repeated heating of PNA samples does not affect their activity.

12. Observe the optical density at $260 \mathrm{~nm}$ (typically a 1:500 dilution).

Synthesis of Modified Oligonucleotides and Conjugates

\subsubsection{5}


13. Determine the concentration using the following equation:

$$
c(\mathrm{mM})=\left(A_{260} \times 30 \mathrm{ng} / \mu \mathrm{L} \times 500\right) / \mathrm{mol} \text {. wt. }
$$

where $30 \mathrm{ng} / \mu \mathrm{L}$ is the extinction coefficient, 500 is the dilution factor, and mol. wt. is the molecular weight of the PNA.

Alternatively, the concentration can be determined as a cumulative function of the extinction coefficients of the PNA monomers. While more time-consuming, the latter method is more accurate since it is based on the extinction coefficient for a specific PNA sequence. The authors have found that it yields concentrations that vary by as much as $50 \%$ to $60 \%$ from those derived from use of the standard conversion factor. To determine the concentration using extinction coefficients, add up the extinction coefficients for the PNA sequence $[A=13.7, C=6.06, G=11.7, T=8.6, \mathrm{~mL} / \mu \mathrm{mol}(\mathrm{cm})]$. Calculate the $O D_{260} \mathrm{~mL}$ of crude PNA and divide by the total extinction coefficient to obtain the millimolar concentration.

Determination of melting temperature provides a useful functional analysis for PNAs. A clean melting curve ensures that the PNA is present in solution at the anticipated concentration and can hybridize to its target sequences. The $T_{m}$ value also provides important information for optimizing annealing conditions and interpreting results.

\section{COMMENTARY}

\section{Background Information}

Peptide nucleic acids (PNAs; Nielsen et al., 1991) are DNA analogs that have a neutral amide backbone (Fig. 4.11.1) and possess physical properties that differ from those possessed by nucleic acids with traditional phosphodiester or phosphorothioate backbones. Although they hybridize with high affinity to DNA and RNA according to normal WatsonCrick base-pairing rules (Egholm et al., 1993), the neutral backbone eliminates the electrostatic repulsion that characterizes the hybridization of DNA and RNA strands. PNA hybridization to single-stranded DNA or RNA occurs with high affinity, and hybridization to duplex DNA is characterized by an outstanding potential for strand invasion (Smulevitch et al., 1996; Lohse et al., 1999; Nielsen, 2001). The absence of a negatively charged backbone also reduces the likelihood that PNAs will associate with cellular proteins (Hamilton et al., 1996) and generate misleading phenotypes. Another difference relative to DNA or RNA is that the strength of PNA hybridization is independent of salt concentration.

Given the many nucleic acid derivatives available, why should researchers consider using PNAs? PNAs possess distinctive chemical properties that confer numerous favorable properties, including high-affinity binding, rapid rates of hybridization, efficient strand invasion, resistance to digestion by nucleases and proteases, and low propensity to bind to proteins. PNAs do not spontaneously enter cultured cells, but can be introduced through simple transfection protocols (Hamilton et al.,
1999; Herbert et al., 1999; Braasch and Corey, 2001; Doyle et al., 2001). PNA synthesis is efficient and versatile and PNAs can be readily purified by HPLC and characterized by mass spectral analysis. Most PNAs will be less soluble than DNA or RNA, but lower solubility can be overcome by adjusting the $\mathrm{pH}$ of stock solutions or by heating PNA solutions prior to use. Learning how to obtain and work with PNAs is not trivial, but the power of PNA recognition amply justifies the effort for many applications. Advantages and disadvantages of PNAs are summarized in Table 4.11.1.

PNAs can also be purchased directly from Applied Biosystems. Other vendors throughout the world have been licensed to sell PNAs. Currently, international vendors include Nippon Flour Mill, Omgen, Sawady Technologies, and OSWEL-University of Southhampton. Applied Biosystems can supply information for the vendor most convenient to a particular laboratory. Obtaining PNAs from commercial sources will probably be a less expensive option for laboratories that require a limited number of PNAs on a $2-\mu \mathrm{mol}$ scale, especially if the laboratories do not have experience making peptides.

\section{Critical Parameters}

The solid-phase synthesis of PNAs uses protocols similar to those developed for peptide synthesis and the physical properties of PNAs are more similar to hydrophobic peptides than to DNA or RNA. PNA solubility at neutral pH is relatively low, and PNAs tend to aggregate upon storage. These properties can pose prob- 
Table 4.11.1 Advantages and Disadvantages of Peptide Nucleic Acids

\begin{tabular}{ll}
\hline \multicolumn{1}{c}{ Advantages } & \multicolumn{1}{c}{ Disadvantages } \\
\hline $\begin{array}{l}\text { Synthesis by standard peptide synthesis } \\
\text { protocols }\end{array}$ & Less soluble than DNA or RNA \\
$\begin{array}{l}\text { Easy to derivatize at N or C terminus } \\
\text { High-affinity hybridization }\end{array}$ & $\begin{array}{l}\text { Tendency for some sequences to aggregate } \\
\text { Requires carrier DNA and lipid for delivery } \\
\text { into mammalian cells or attachment to import } \\
\text { peptides }\end{array}$ \\
High rates of hybridization & $\begin{array}{l}\text { Exceptional potential for strand invasion } \\
\text { Low potential for binding to proteins that bind } \\
\text { negatively charged polymers }\end{array}$ \\
\hline
\end{tabular}

lems for researchers unfamiliar with PNAs, and methods for obtaining useful concentrations of soluble PNA will be discussed below. The authors have not found poor solubility to be a major barrier to experiments with PNAs since the solubility of PNAs is a $\mathrm{pH}$-dependent property. PNAs are soluble to high concentrations at $\mathrm{pH} \leq 5.0$, and usually remain soluble at neutral $\mathrm{pH}$ when diluted to lower concentrations or when $\mathrm{pH}$ is slowly adjusted upward. A substantial advantage is that PNAs can be readily derivatized by peptides or other groups that can form a covalent linkage with the free amino terminus.

For automated synthesis, proper maintenance of the instrument is essential. One common problem is clogged lines that can lead to reduced flow rate. Line-filters should be replaced monthly on all internal bottles to ensure that the flow of reagents is consistent for all syntheses. When bottles are removed or placed on the instrument, the open lip of each bottle should be wiped with a clean Kimwipe to prevent cross-contamination of bottles and reduce wear on the O-ring seals. The O-ring seals should be replaced every 3 months regardless of any appearance of physical stress.

\section{Troubleshooting}

A PNA that has been correctly synthesized will usually give rise to predominantly one peak by HPLC purification. However, PNAs that possess a high likelihood for forming internal structure may give rise to multiple peaks. The potential for multiple peaks can be lessened by heating the HPLC column at $55^{\circ} \mathrm{C}$. If mass spectral analysis shows that the major peaks share a single predominant product, multiple peaks may not be a cause for concern. The most common problem with PNA synthesis is formation of truncated products.

One cause of failed syntheses is use of reagents that are contaminated with water, leading to a reduction in coupling efficiency. PNA monomer and activator should be routinely dried in vacuo prior to synthesis. The authors also use fresh bottles of solvent, $\leq 14$ days old.

Alternatively, failed sequences could arise due to problems with automated synthesis or human error during manual synthesis. If truncated products are formed, the HPLC will show a series of peaks with shorter retention times than would be expected for full-length product.

Another problem is failure to fully cleave protecting groups after completion of synthesis. Since the protecting groups are hydrophobic, a failure to cleave them results in products with longer retention times after RP-HPLC. PNAs that have this problem will appear to have at least two peaks upon HPLC analysis and can be salvaged by a second treatment with TFA and $m$-cresol. Retention of FMOC groups can also be confirmed by observation of a mass of 222 atomic mass units (amu) greater than expected. To avoid this problem, the authors use TFA within 1 month of first opening bottles.

The solubility properties of PNA are discussed above. PNA oligomers have different solubility properties than analogous DNA oligonucleotides and will often be less soluble. Dissolving PNA stocks at $\mathrm{pH} \leq 5.0$ and heating solutions prior to use should alleviated this. The authors routinely check the concentration of PNA stocks by monitoring their absorbance at $260 \mathrm{~nm}$ to ensure that the expected concentration is being maintained.
Synthesis of Modified Oligonucleotides and Conjugates

\subsubsection{7}




\section{Anticipated Results}

The authors' laboratory has made several hundred PNAs. When the Expedite synthesizer is working properly and when all reagents are fresh and dry, an excellent yield of the desired product is almost invariably achieved. Crude material before purification contains $\geq 50 \%$ desired product, while HPLC-purified product contains $>90 \%$. PNAs and PNA-peptide conjugates that require as many as 40 coupling steps can be synthesized in good yield. It is possible to synthesize PNAs with long runs of purines or PNAs that are self-complementary as long as critical coupling steps are repeated. PNA synthesis is actually easier than peptide synthesis because the "personalities" of only four, rather than twenty, monomers are involved in determining coupling efficiency. With care, almost any PNA can be obtained.

\section{Time Considerations}

Preparing the reagents for automated or manual PNA synthesis requires 1 to $2 \mathrm{hr}$. Automated PNA synthesis can be completed within hours, depending on the length of the PNA. Manual synthesis should take no more than 1 $\mathrm{hr}$ per cycle depending on whether a capping step is performed. Deprotection and purification can be accomplished in 1 day. HPLC analysis and purification should take 2 to $3 \mathrm{hr}$ combined. MALDI-TOF-MS should require no more than $30 \mathrm{~min}$.

\section{Literature Cited}

Braasch, D.A. and Corey, D.R. 2001. Synthesis, analysis, purification, and intracellular delivery of peptide nucleic acids. Methods 23:97-107.

Doyle, D.F., Braasch, D.A, Simmons, C.G., Janowski, B.A., and Corey, D.R. 2001. Intracellular delivery and inhibition of gene expression by peptide nucleic acids. Biochemistry 40:53-64.

Egholm, M., Buchardt, O., Christensen, L., Behrens, C., Freier, S.M., Driver, D.A., Berg, R.H., Kim, S.K., Norden, B., and Nielsen, P.E. 1993. PNA hybridizes to complementary oligonucleotides obeying the Watson-Crick hydrogenbonding rules. Nature 365:566-568.

Goodwin, T.E., Holland, R.D., Lay, J.O., and Raney, K.D. 1998. A simple procedure for solid-phase synthesis of peptide nucleic acids with N-terminal cysteine. Bioorg. Med. Chem. Lett. 8:22312234.

Hamilton, S.E., Iyer., M., Norton, J.C., and Corey, D.R. 1996. Specific and nonspecific inhibition of RNA synthesis by DNA, PNA and phosphorothioate promoter analog duplexes. Bioorg. Med. Chem. Lett. 6:2897-2900.
Hamilton, S.E., Simmons, C.G., Kathriya, I., and Corey, D.R. 1999. Cellular delivery of peptide nucleic acids and inhibition of human telomerase. Chem. Biol. 6:343-351.

Herbert, B.-S., Pitts, A.E., Baker, S.I., Hamilton, S.E., Wright, W.E., Shay, J.W., and Corey, D.R. 1999. Inhibition of telomerase in immortal human cells leads to progressive telomere shortening and cell death. Proc. Nat. Acad. Sci. U.S.A. 96:14726-14281.

Lohse, J., Dahl, O., and Nielsen, P.E. 1999. Doubleduplex invasion by peptide nucleic acid: A general principle for sequence-specific targeting of double-stranded DNA. Proc. Natl. Acad. Sci. U.S.A. 96:11804-11808.

Mayfield, L.D. and Corey, D.R. 1999. Automated synthesis of peptide nucleic acids (PNAs) and peptide nucleic acid-peptide conjugates. Anal. Biochem. 268:401-404.

Nielsen, P.E. 2001. Targeting double-stranded DNA with PNA. Curr. Med. Chem. 8:545-550.

Nielsen, P.E., Egholm, M., Berg, R.H., and Buchardt, O. 1991. Sequence-selective recognition of double stranded DNA by a thymine-substituted polyamide. Science 254:1497-1500.

Norton, J.C., Waggenspack, J.J., Varnum, E., and Corey, D.R. 1995. Targeting peptide nucleic acid protein conjugates to structural features within duplex DNA. Bioorg. Med. Chem. 3:437-445.

Simmons, C.G., Pitts, A.E., Mayfield, L.D., Shay, J.W., and Corey, D.R. 1997. Synthesis and membrane permeability of PNA-peptide conjugates. Bioorg. Med. Chem. Lett. 7:3001-3007.

Smulevitch, S.V., Simmons, C.G., Norton, J.C., Wise, T.W., and Corey, D.R. 1996. Enhanced strand invasion by oligonucleotides through manipulation of backbone charge. Nature Biotech. 14:1700-1704.

Zhang, X., Ishihara, T., and Corey, D.R. 2000. Strand invasion by mixed base PNAs and PNApeptide chimera. Nucl. Acids Res. 28:3332-3338.

\section{Internet Resources}

http://www.appliedbiosystems.com/ds/pna.taf

Ordering information and bibliography.

http://www.horizonpress.com/gateway/pna.html

Links to PNA-related sites.

http://www.isogen.nl/pna.html

PNA synthesis provider in The Netherlands.

http://www.bostonprobes.com

Supplier of PNA diagnostic probes.

Contributed by Dwaine A. Braasch,

Christopher J. Nulf, and David R. Corey

University of Texas Southwestern Medical

Center at Dallas

Dallas, Texas
Synthesis and Peptide Nucleic Acids

4.11.18 